\title{
QUANTIFYING THE IMPACT OF URBAN INFILL ON THE URBAN HEAT ISLAND EFFECT - A CASE STUDY FOR AN ALTERNATIVE MEDIUM DENSITY MODEL
}

\author{
P. Helmholz ${ }^{* 1}$ D. Bulatov ${ }^{2}$, B. Kottler ${ }^{2}$, P. Burton ${ }^{3}$, F. Mancini ${ }^{1}$, M. May ${ }^{1}$, E. Strau $\aleph^{2}$, M. Hecht ${ }^{2}$ \\ ${ }^{1}$ Curtin University, GPO Box U1987, Perth WA 6845, Australia (petra.helmholz; francesco.mancini@curtin.edu.au; \\ mariebeth.may@postgrad.curtin.edu.au) \\ ${ }^{2}$ Fraunhofer Institute of Optronics, System Technologies and Image Exploitation (IOSB), Gutleuthausstr. 1, 76275 Ettlingen, \\ Germany (dimitri.bulatov; benedikt.kottler; eva.strauss, marko.hecht@iosb.fraunhofer.de) \\ ${ }^{3}$ Open Space Design Australia (OSDA), Bassendean, 6054, WA (phil@osda.com.au)
}

\section{Commission IV}

KEY WORDS: Urban infill, urban heat islands, sensor, temperature, urban planning

\begin{abstract}
Urban Heat Islands (UHIs) impact the quality of life in many urban centres. Metropolitan areas of Australian cities and urbanised regional centres, in particular, show vulnerability towards UHIs due to challenging climatic conditions and the model of greater subdivision of established properties whereby backyards and mature trees are replaced with more residential dwellings and sealed areas. The measurements for the UHI mitigation, such as imposing reforestation, employing sustainable and medium density housing build form typology must be quantified. Simulation-based identification and mitigation of UHIs can be used for planning decisions. There are several advantages to use simulations. For instance, alternative subdivision design, building design and the placement of trees and other measures is only required in the digital twin. Experimenting with the digital twin saves resources and maximises the outcome by being able to quantify the heat reduction. The aim of this work is to quantify the impact of the current urban infill methods on UHI. We focus on an area that has gone through a process of re-subdivision that is bounded by roads within Perth, Western Australia. For the same area we propose an alternative design with an urban infill model based on a medium density housing guidelines with an improved green space allocation. While the current model shows significant effects of UHIs we could mitigate those effects in the alternative proposed model. In our simulation the hottest surfaces are roads with a temperature of approximately $45^{\circ} \mathrm{C}$ at $2 \mathrm{PM}$ in the afternoon. In the current model close to $100 \%$ of the road surfaces reach this temperature. In contrast, in the alternative model only $45 \%$ of all road surfaces reach this temperature, significantly reducing the impact of UHI for pedestrian walking close by to those roads. At $2 \mathrm{PM}$, the most frequent temperature of all surfaces for the current model is around $44^{\circ} \mathrm{C}$ while the alternative's model the most frequent temperature is approximately $28^{\circ} \mathrm{C}$ - a difference of $16^{\circ} \mathrm{C}$.
\end{abstract}

\section{INTRODUCTION}

In the present century, for the first time in history, the urbanised population of the world has overtaken the rural population. Cities are growing and green space areas on the fringe of cities are constantly being consumed by built form. Using MODIS and SPOT satellite data, (Chen et al., 2017) showed that, with progressing urbanisation, the land surface temperature increases, too. (Liang et al., 2021) could quantify these temperature changes using Landsat imagery. The effect of increased temperatures in urban settlements is called Urban Heat Islands (UHI).

Leaving aside global climate change (which, according to some studies, can negatively influence UHI, Mahmuda and Webb, 2016), there are the two main contributing factors to UHI in Australia. Firstly, urban sprawl means claiming of bush land and market gardens for more housing estates on the fringe of major cities and town sites. Secondly, infill is the well-known approach to increase the population density in already established urban areas. It is often caused by further subdivision of established properties and regularly replaces backyards and mature trees with more residential dwellings and sealed areas. On the one hand, infill brings advantages in terms of proximity, walkability, and critical mass to support efficient public bus transport, active transport (biking), shops, kindergartens, health services, pharmacies etc. On the other hand, density needs to be well- managed and designed, because otherwise, the effects of UHI will be even greater than that caused by urban sprawl.

In Western Australia (WA), current infill outcomes are understood to lead to a reduction of green, vegetated, and porous surfaces, transforming them to sealed surfaces. This has negative consequences contributing to the effects of UHI. To be able to advise on policies with decision-makers, it is important to understand the impact of the current method of urban infill on UHI, more specifically, to quantify the temperature change.

On a local level, the green spaces within cities are shrinking to give way to more buildings, which leads to further accelerating the effect of UHI. In this context, studies investigate the comparison between the climatic conditions (heat) in various types of urban areas, including green open spaces, exposed open urban squares, and road corridors near such sites. Indeed, (Hwang, et. Al., 2015) showed that micro-scale thermal performance could vary even within a distance of $10 \mathrm{~m}$.

Most local scale research has focused on single aspects, such as shading from trees, surface colours and materials, radiation, and a flux of surfaces. Nevertheless, a better understanding of the interrelationship of these aspects regarding their impact on UHI is a challenge. For instance, at this local level, the use of satellite data is insufficient as single trees or other urban features cannot

\footnotetext{
* Corresponding author
} 
be modelled. Instead, urban planners often use scaled models, e.g. consisting of an array of concrete cubes (Park et al., 2012) or expansive sensor systems (Cohen et al., 2012), or low-cost sensors (Williams, et al., 2019), to quantify the urban heat impact and its different factors.

An alternative is to run computer simulations to measure urban heat. Several researchers (Taleghani et al., 2014; Acero and Herranz-Pascual, 2015; Lobaccaro and Acero, 2015; Lee and Mayer, 2018) used the ENVI-met model to investigate heat island effects. ENVI-met calculates the state of the atmosphere by combining the influence of buildings, vegetation, surfaces' characteristics, soils and climatic contour conditions. This program is a three-dimensional microclimate model designed to simulate the surface, plant and air interactions in an urban environment. ENVI-met is generally used with a typical spatial resolution of 0.5 to $10 \mathrm{~m}$. The software works with voxels, which makes it difficult to consider roof shapes in residential areas.

The aim of this work is to quantify the impact of the current urban infill methods on UHI. We focus on a residential block sized area bounded by roads within Perth, WA. Perth's climate is classified as warm temperate, Mediterranean. The city is currently going through a phase of high levels of urban infill.

We analyse the UHI effect for two scenarios of this area:

1. A precinct that has incurred re-subdivision and residential urban infill as per current state government policies referred to as Current Model.

2. The same precinct area with an urban infill model based on the new medium density housing policy with improved green space allocation as promoted by the Department of Planning, Lands and Heritage, WA. - referred to as Alternative Model.

The paper is structured as follows: In the next section, the study area and the data used for the heat simulation is presented. Next, in the methodology section, the workflow for thermal simulation of semantic 3D models (Bulatov et al., 2020) is briefly described together with the metrics allowing for quantification of the urban heat island effect. The results are discussed in the evaluation section before the findings are summarised.

\section{STUDY SITE AND DATA}

The study site used for this research is the Quattro Precinct which was formally known as the Maniana Precinct. It is approximately a 21-hectare subdivision located within the suburb of Queens Park and the municipality of the City of Canning in Perth, WA (Figure 1). The site is in the South Eastern suburbs of the metropolitan area. The closest train station is Queens Park approximately $1.2 \mathrm{~km}$ away. Neighbouring the site to its East there is a large parkland area that provides a sports field and natural areas.

The Maniana Precinct was designed in 1953 by Margaret Feilman (1921- 2013) (Menck, 2014) who was the first dual qualified architect and town planner in the state of Western Australia (Ethell, 2013). The new subdivision was to encompass 'Garden City' design principals (Menck, 2014), connecting its residents to the landscape and the neighbouring employment centre of Welshpool. The project, to provide state government housing following World War II, was constructed in 1954- 1955 where 300 timber framed homes were built in 31 weeks (Menck, 2014)

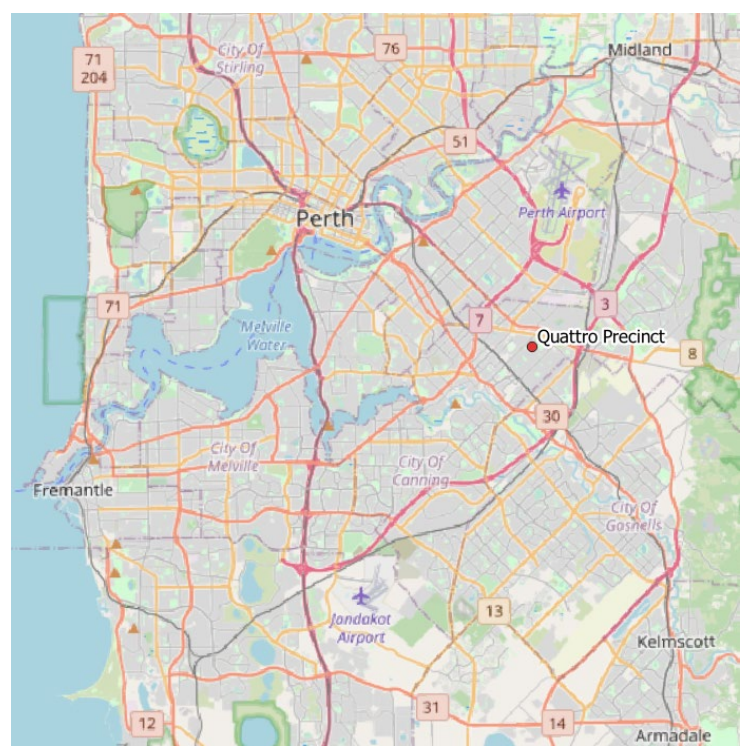

Figure 1: The location of the Quattro Precinct within Perth (data source: Open StreetMaps (www.osm.org)).

Following extending and significant refurbishment of the dwellings between 1978 and 1980 to extend the life of the precinct as it was, the state government of WA decided in 2003 to demolish it and make over the precinct to reduce the concentration of state government owned housing in the precinct and allow a large portion of the redeveloped area to be made available for the public to purchase and build private housing on.

A masterplan was developed by a private town planning consultancy comprising of lot sizes of between 350sqm and $600 \mathrm{sqm}$. The outcome of this masterplan redevelopment is the model presented today as our Current Model.

Using aerial imagery of the current model its was easy to visually observe the large extent to which individual houses occupy a significant footprint of each individual property lot (Figure 2). These large footprints leave little available room for trees or vegetation in general on each lot and can result in owners paving a large extent of there outdoor private space.

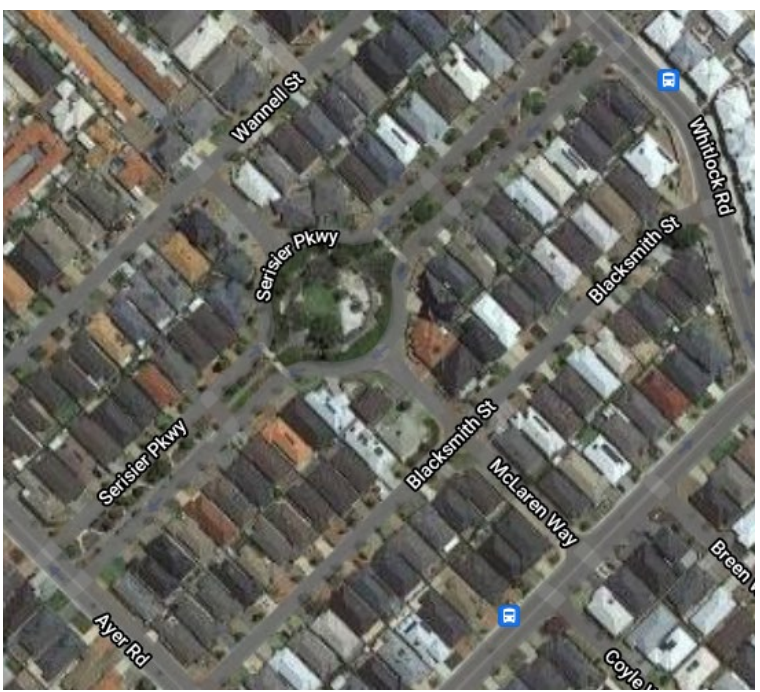

Figure 2: Aerial image of the sample area of the current Quattro Precinct subdivision (data source: GoogleMaps). 


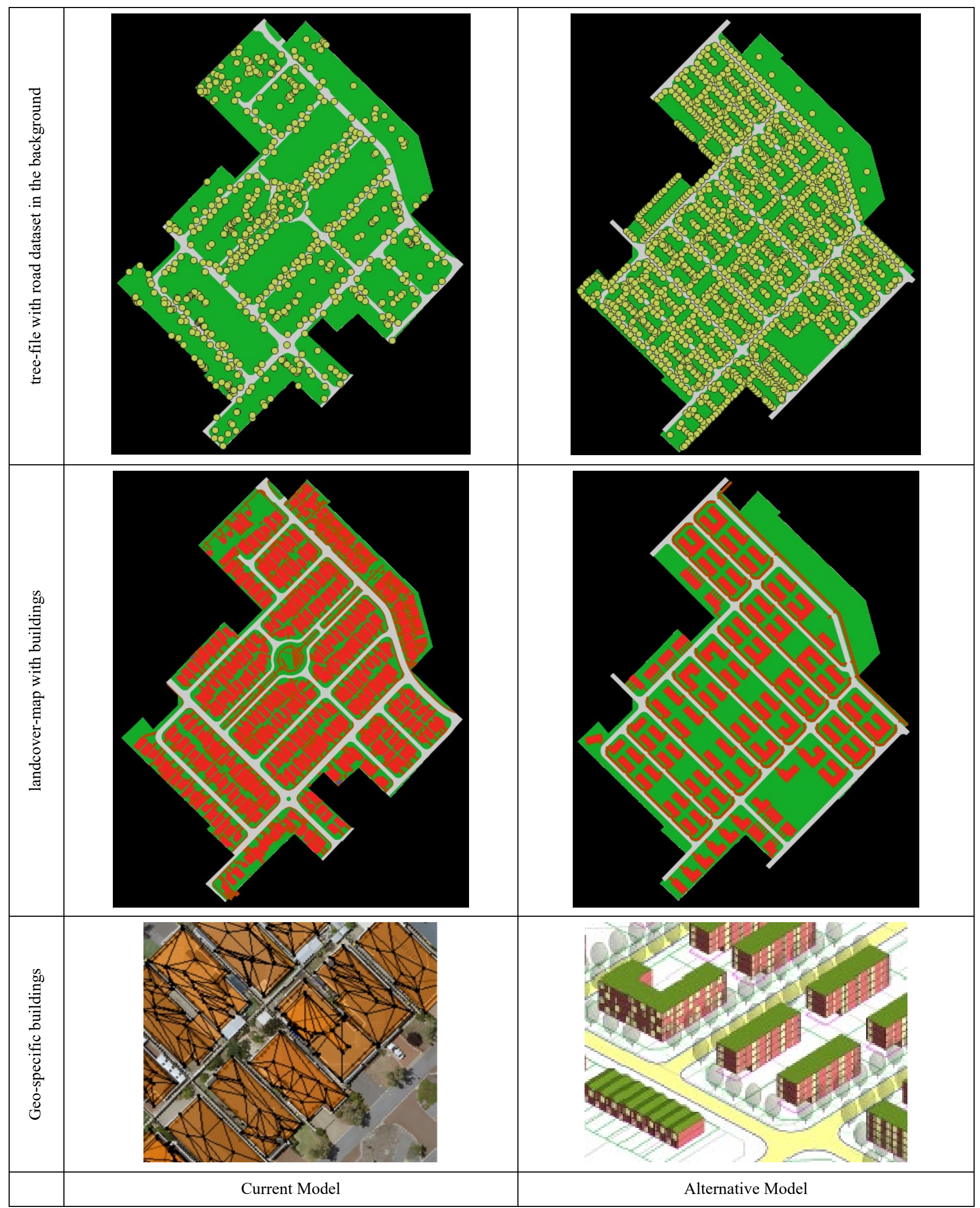

Figure 3: The tree locations (top row), the landcover map (middle row) and a fragment showing of geo-typical buildings (bottom row) for the Current Model (left) and the Alternative Model (right). The colours for the landcover map are green (grass), red (building), brown (paving/soil) and grey (road/asphalt). In the bottom left image, the buildings overlay the ortho-image, which is not available for future scene. 
From these observations an Alternative Model set of principles were developed based on the medium density code that would allow for greater population density. These are:

- Limit building footprints to a maximum of $50 \%$ of the lot,

- Restrict car parking to within the building footprint- at ground level below residences,

- $\quad$ Maximise realistic street tree planting spacings and canopy coverage.

- Ensure $10 \mathrm{~m}$ minimum open space between apartment buildings (the majority have 12 metres of space between rear and side facades).

- Allow for outdoor space surrounding buildings for gardens of various types.

- Allow for community garden spaces for the cultivation of food and social interaction and additional food productive tree species that will contribute to tree canopy cover.

- Allow for a variety of tree size and canopy spread.

- Promote shaded walkable links.

- Promote and allow for foreground and broader views over a vegetated urban landscape.

Information about the dwellings and the tree canopy area for both models are presented in Table 1. Overall, the alternative model provides over three times as many dwellings and also nearly three times of the tree canopy.

\begin{tabular}{|l|c|c|}
\hline & Current & Alternative \\
\hline $\begin{array}{l}\text { Number of } \\
\text { dwellings/apartments }\end{array}$ & 329 & 1188 \\
\hline Dwelling density & $\begin{array}{c}15.7 \\
\text { dwellings/ha }\end{array}$ & $\begin{array}{c}56.6 \\
\text { dwellings/ha }\end{array}$ \\
\hline Number of trees & 619 & 1999 \\
\hline Total tree coverage & $0.74 \mathrm{ha}$ & $5.5 \mathrm{ha}$ \\
\hline Mean tree coverage area & $12.0 \mathrm{sqm}$ & $27.5 \mathrm{sqm}$ \\
\hline
\end{tabular}

Table 1: Density and tree coverage information of the Current Model and the Alternative Model.

For each model, the following information are required for the input of the heat simulation:

- A raster file containing the landcover classes - referred to as landcover-map.

- A point-vector file with the location of trees- referred to tree-cadastre.

- Vector file containing the building outlines/footprints referred to as building-cadastre.

- Building model library, a library of geo-specific buildings.

The landcover map (i.e. materials and built form covering the ground surface) contains the classes asphalt, paving, building, and water. The number of classes is limited in our simulations and a separation of irrigated and non-irrigated grass is not considered. For instance, in the current model it was identified that there were significant areas of non-irrigated grass which has temperature characteristics closer to soil then irrigated grass in summertime. As the effect is seasonal and to make the models comparable, a more detailed split between the classes was not performed. The landcover map is converted into a triangled mesh in $3 \mathrm{D}$ using the adaptive triangulation method (Pajarola, R. (2002)) whereby for simplicity, the third coordinate is always chosen to be zero. This triangle mesh is semantic, because every triangle has its assigned class, and represents our ground model.
The tree cadastre contains the location of all trees including their height and canopy spread. For the current model, a cadastre was provided by the City of Canning which was updated by manually digitising missing trees using an ortho-image of $6 \mathrm{~cm}$ resolution. During the manual digitisation, the tree cadastre was used to estimate the trees' heights and crown diameters by comparing missing trees to those included into the existing database. In addition, the approximation of the height was supported by displacement and shadows present in the ortho-image. For the alternative model, appropriate tree heights and crown diameters were chosen trying to optimise the tree coverage to building ratio. From every tree position, we extended the mesh with a tree-like structure, which has been created using a higher-order bivariate function.

The building cadastre contains further information about the overall building height, the roof material (tiles or Colorbond), the roof colour and a reference to the model type of the building. Tiles are known to be either clay or concrete in materiality and Colorbond is a brand of manufacturer painted corrugated sheet steel and often a term used generically to describe any painted corrugated sheet steel roofing. For the current model, an existing building database provided by the City of Canning has been updated using the $6 \mathrm{~cm}$ ortho-image. Most buildings in the Current Model are single story detached houses with a maximum roof ridge height of approximately $6 \mathrm{~m}$. The most common roofing material is tiles (195 dwellings) compared to 134 Colorbond roofed dwellings. The buildings in the Alternative Model are designed to meet the guidelines of the new medium density policy. The Alternative Model has overall two main building styles - multi-story buildings with a height of $13.2 \mathrm{~m}$ using a Colorbond roofing material and town houses with a height of $7.8 \mathrm{~m}$ using tiles as roofing material.

The building model library contains 3D building models (LOD3) which can be found in the current and alternative model. For the current model, the ortho-image has been used to design the building model; more details are available in (Bulatov at al., 2021). For the alternative model, eight $3 \mathrm{D}$ designed models have been created.

All datasets are presented in Figure 3. An ortho-image is additionally provided for visualization and can only be used, of course, for the current model only.

\section{METHODOLOGY}

\subsection{Heat Simulation}

As mentioned before, the ground model, 3D buildings and vegetation (trees) are converted into triangle meshes. Thus, for each scenario, a semantic 3D city model is created based on the the landcover-map, tree and building cadastres, and the building model library. Each triangle, besides its geometrical property, has a unique material. The physical properties of all materials are stored in a library and assigned to each triangle of the mesh to formulate terms for heat conduction, heat radiation and convection. These terms depend on the unknown surface temperature and, according to the Heat Balance Equation, their sum is directly proportional to its temporal derivative. The resulting partial differential equation is solved using Euler forward integration method. More details are provided in (Kottler et al., 2019). 


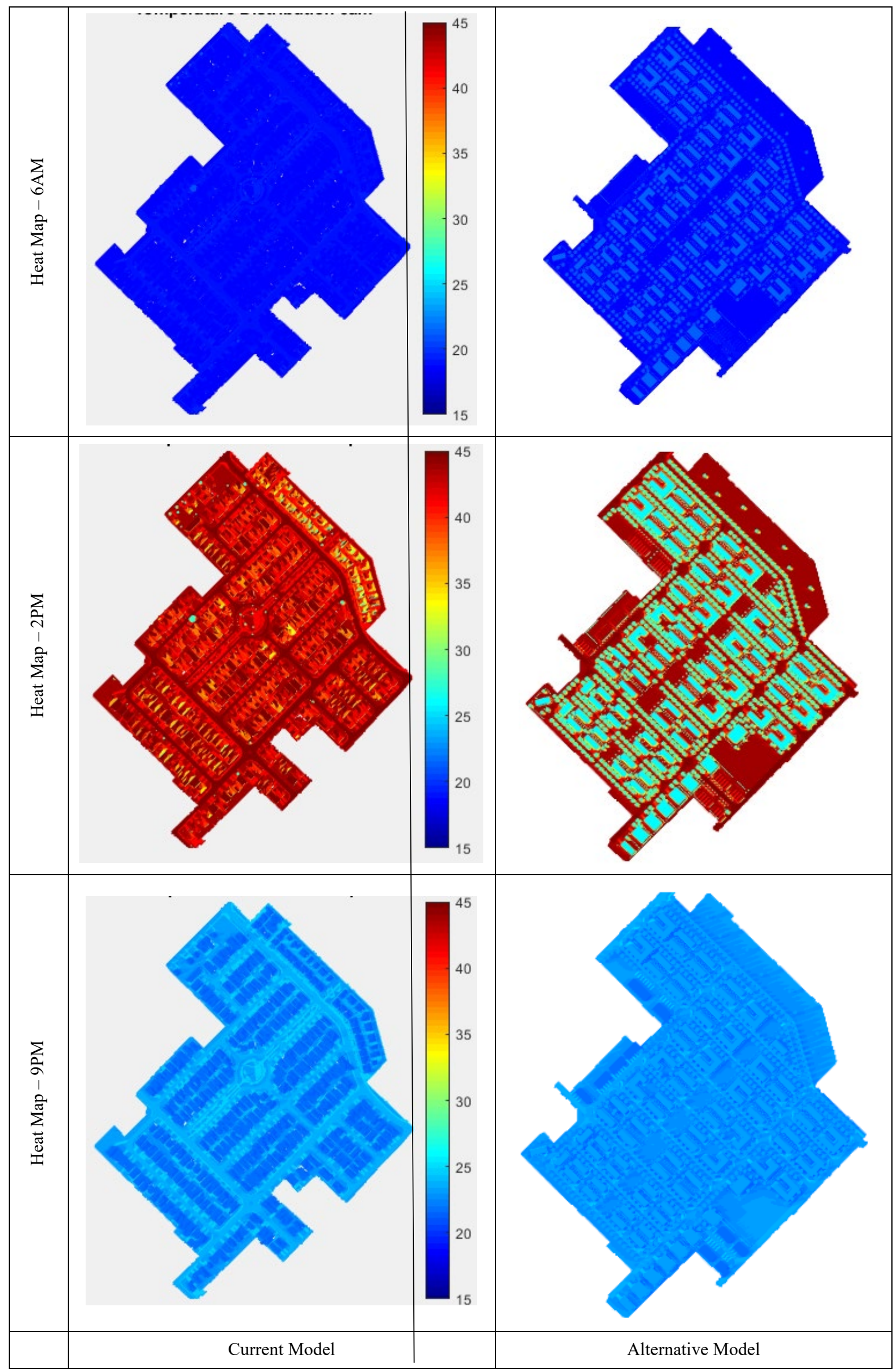

Figure 4: Results of the heat simulation of the current (left) and alternative (right) model. 
The initial values for the triangles' temperatures are given by the temperature of air at night. Due to thermal inertia and therefore a strong dependency on the initial values of temperature, a period of three days was firstly simulated. The temperatures presented in this paper are related to day four, so the day after the initiation of the model. The air temperature used for the simulation of day four is shown in Figure 5. The day would be ranked as a mild summer's day in Perth.

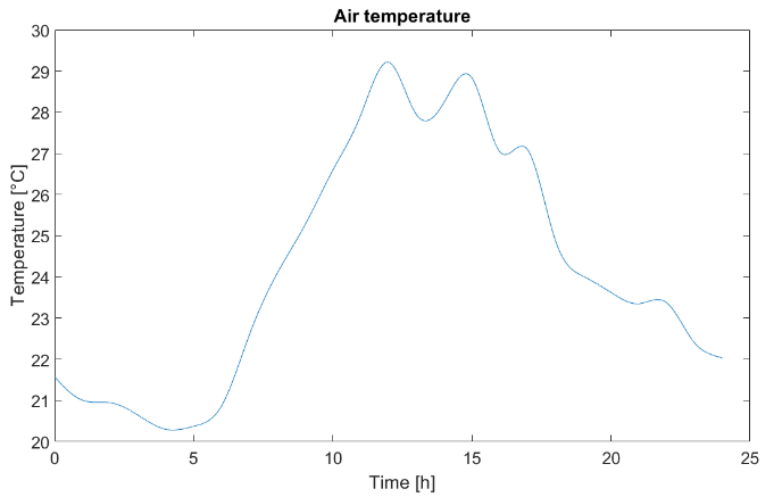

Figure 5: Air temperatures used for the simulation.

Applying the heat balance equation consisting of terms for heat conduction, convection and radiation we can compute the surface temperature as a function of time of each triangle in the mesh (Bulatov et al., 2020).

\subsection{Derived measures}

Besides a visual inspection, quantitative measures are derived to analyse the UHI. For breaking down the measurements to assess the scene design, we use statistical measures, i.e. average temperature of all surfaces and specifically, road surfaces. The road surfaces are of particular interest as this is the environment, in which pedestrians walk cyclists ride, and thus are most capable to notice the impact of Urban Heat Islands. For visualisation, we use histograms.

Those quantitative measures include:

- Mean temperature plots of different surfaces classes

- Temperature frequency for specific classes.

\section{EVALUATION}

\subsection{Visual Inspection}

The results of the heat simulation are shown in Figure 4. The left and right columns show the results related to the current and the alternative model, respectively. The three time points chosen are $6 \mathrm{AM}$ in the morning (top row), 2PM in the middle of the heat period (middle row), and 9PM at the end of the day (bottom row). These time points were selected to show the development from the morning to the peak temperature of the day (around 2PM) as well as considering the temperatures at night times 9PM as this is where the UHI are mostly noticeable because the urban environment cannot cool down.

At 6AM, all surfaces in the current model have approximately the same temperature and are around the minimum overnight temperature. The surfaces have not started heating up. In contrast, the alternative model shows some areas above the minimum temperature. These areas are the trees, which tend not to cool down below $20^{\circ} \mathrm{C}$ because as living organisms, they are supposed to maintain their core temperature constant.

At $2 \mathrm{PM}$, during the hottest time of the day, it is easily visible that the $29^{\circ} \mathrm{C}$ air temperature leads to much higher temperature for several surfaces. The absence of large roadside trees leads to higher temperatures along road corridors in the current model compared to the alternative model. The coolest areas in the alternative model are by far the trees followed by tile roofs. Missing trees in the current model make the current model a "much hotter" space. Please note that while tiled roofs need longer to warm up, those areas are the hottest areas at night-time radiating heat long after sunset.

At 9AM, the hotter tile roofs compared are visible in the current and alternative model. In contrast, the Colorbond roofs cool down much quicker, as can be observed in both models. In the current model, it is also visible that the road surfaces are, on average, hotter than in the alternative model due to the higher shading of road surfaces in the alternative model during the day.

\subsection{Temperature plots of the different surface classes}

The temperature plots of the different surfaces are presented in Figure 6. Overall, the hottest surface is the roads followed by grass. The next classes are soil followed by buildings. The coolest class (beside water) are trees. While trees are the coolest during the day, they are the warmest during the night.

The orange lines indicate when the road surface reaches $25^{\circ} \mathrm{C}$ in the current model. It is visible that the current model reaches the $25^{\circ} \mathrm{C}$ mark approximately $45 \mathrm{~min}$ before the alternative model. Therefore, in the alternative model, the road temperature stays cooler for longer in the morning compared to the current model. Furthermore, the road surfaces cool down quicker in the alternative model and reach the $25^{\circ} \mathrm{C}$ mark approximately $30 \mathrm{~min}$ earlier at night-time.

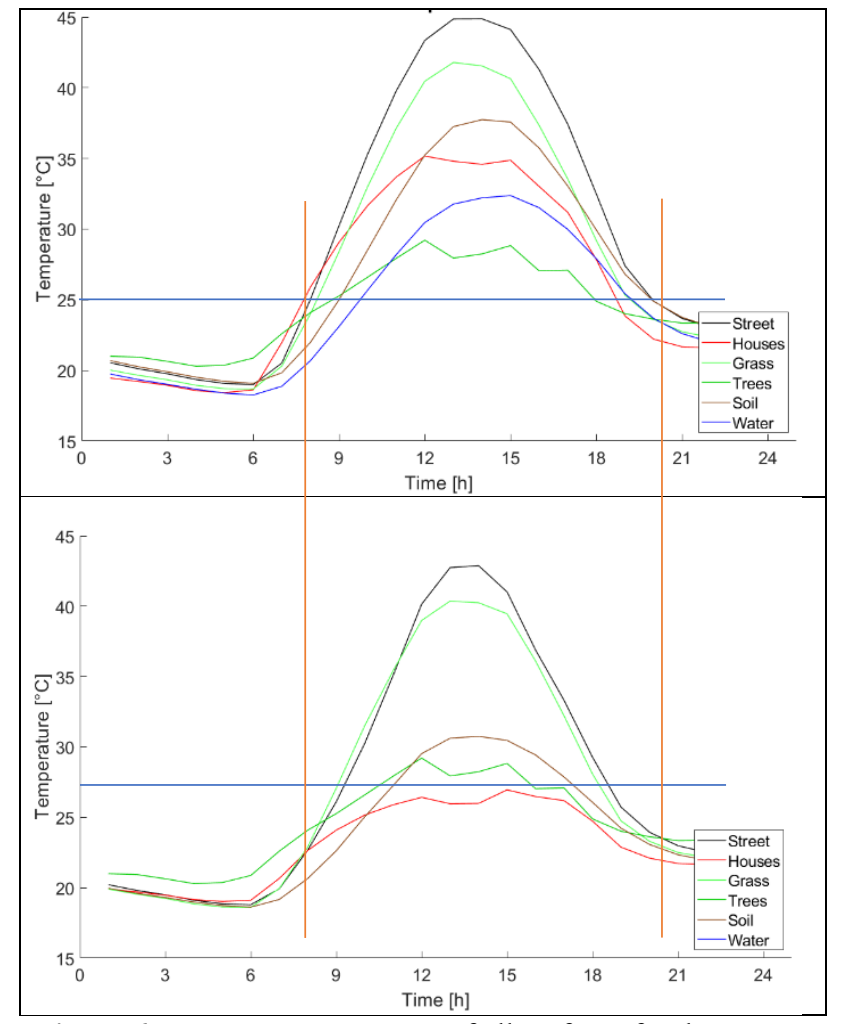

Figure 6: Average temperatures of all surfaces for the current model (top) and alternative model (bottom).

For the rest of the paper we focus on the cumulative heat over all surfaces as they are important to quantify the heat island effect and on road surfaces as those are the areas in which pedestrian get expose to the temperatures. 


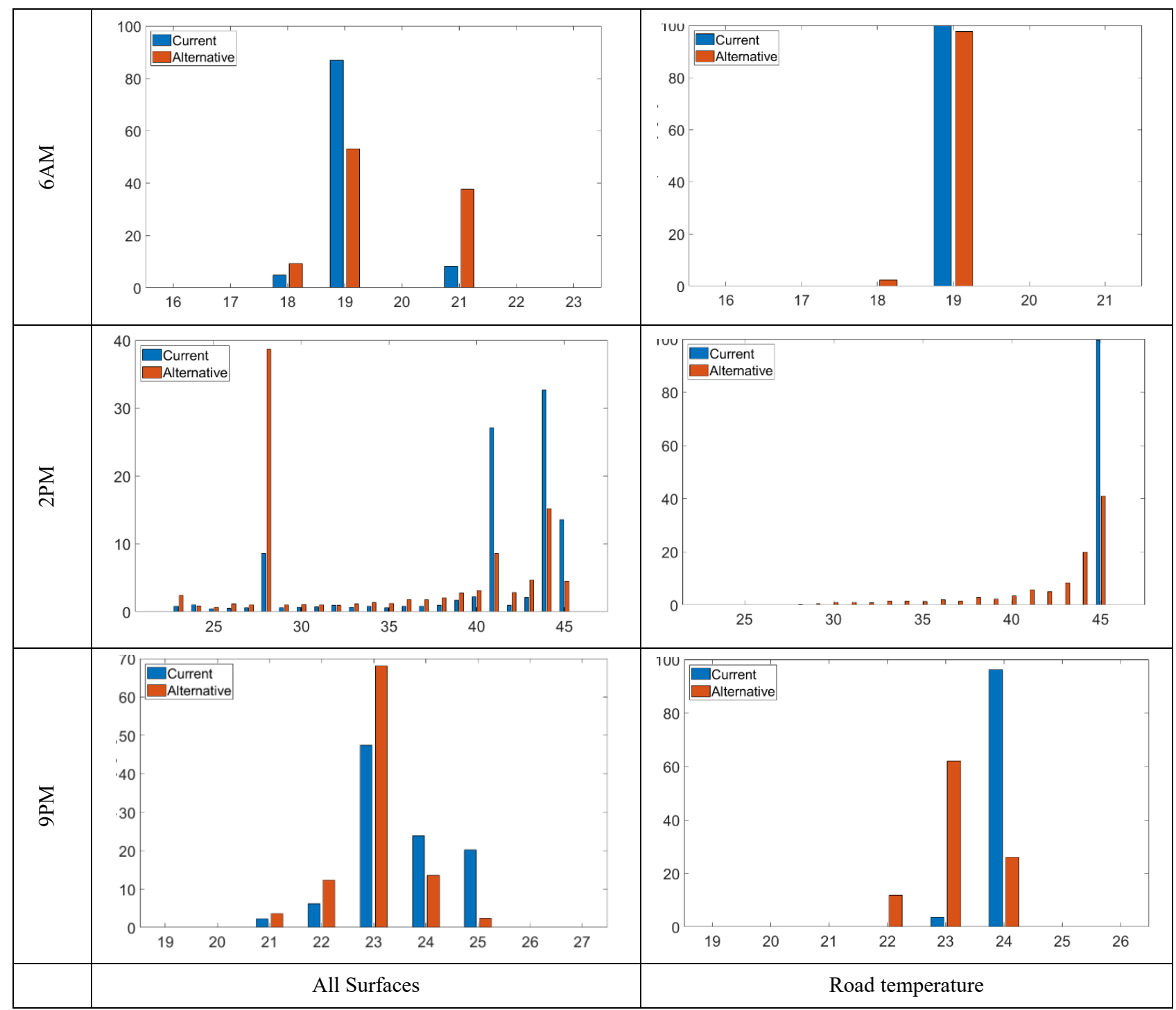

Figure 7: Temperature frequency in percent of all surfaces (left) and of road surface (right) at different time.

\subsection{Overall temperatures}

Figure 7 presents the temperature frequency as a percent of all surfaces (left) and of road surfaces (right) at different time for the current model (blue) and alternative model (red)

In the early morning (6AM) the temperatures over all surfaces of both models are very similar. The most frequent temperature is around $19^{\circ} \mathrm{C}$. In our simulation, the alternative model appears slightly warmer, due to the high number of trees as discussed previously. The situation has changed significantly for the $2 \mathrm{PM}$ plot. The most frequent temperature for the current model is around $44^{\circ} \mathrm{C}$ while the alternative's model most frequent temperature is around $28^{\circ} \mathrm{C}$. This is a different of $16^{\circ} \mathrm{C}$ !

While focusing on the road temperature only (right column of Figure 7), the temperatures of the current and alternative model are comparable in the morning with being around $19^{\circ} \mathrm{C}$. At $2 \mathrm{PM}$, the most frequent temperature for both models is $45^{\circ} \mathrm{C}$. However, the total percentage of the histogram bin corresponding to $45^{\circ} \mathrm{C}$ road surfaces of the current model is close to $100 \%$. In contrast, in the alternative model, this bin takes on only $45 \%$ of all road surfaces. Cooler temperatures are more common due to the shading of the roads by trees. This shading also contributes to a faster cooling of the road surfaces at night-time in the alternative model.

\section{CONCLUSION}

In this paper, we created two semantic models. Firstly, a model of the current Quattro Precinct in its subdivision layout, low-rise (generally single storey) built form, green space allocation and urban tree canopy. This form of urban density is now not uncommon in metropolitan Perth, particularly within already established suburbs and those being developed on the urban fringe. Secondly, an alternative model with the intent of achieving a higher building and population density that aligns with the medium density planning codes, while also providing a higher private (per lot) and public green space allocation and overall urban tree canopy. Applying the thermal simulation of (Bulatov et al.,2020) to both models, we were able to quantify the urban heat for both models.

The current model shows a significant pattern to what is referred to be an "Urban Heat Island", an area which stores heat and 
overall leads to much higher localised temperatures effecting the health and wellbeing of the residents. The alternative model provides not only as many dwellings as the current model, but substantially exceeds it. This model could reduce the effects of the Urban Heat Island while applying an alternative model of medium density-built form in combination with a higher proportional footprint of green spaces allowing for them to contain more trees and space for tree canopy.

In this paper, we show how simulations could be utilised by urban planners, urban designers, landscape architects and architects to benefit urban design and development proposals by quantifying the impact of urban heat with a view to mitigating its effects.

\section{ACKNOWLEDGEMENTS}

We would like to acknowledge and thank the 2020 SPUR Accelerator Program from Landgate which enabled this research and the participation in the conference. Also, David Ford at the City of Canning and the City of Canning for providing data for the paper.

\section{REFERENCES}

Acero, J.A., Herranz-Pascual, K., 2015. A comparison of thermal comfort conditions in four urban spaces by means of measurements and modelling techniques. Building and Environment, 93 (2015) 245-257.

Berghauser Pont, M. B., Stavroulaki, G., Bobkova, E., Gil, J., Marcu, L., Olsson, J., Sun, K., Serra, M., Hausleitner, B., Dhanani, A., Legeby, A., 2019a. The spatial distribution and frequency of street, plot and building types across five European cities. Urban Analytics and City Science, 46(7) 2019, 12261242 .

Berghauser Pont, M. B., Stavroulaki, G., Marcu, L., 2019b. Development of urban types based on network centrality, built density and their impact on pedestrian movement. Urban Analytics and City Science, 46(8) 2019, 1549-1564.

Bulatov, D., Burkard, E., Ilehag, R., Kottler, B., and Helmholz, P., 2020. From multi-sensor aerial data to thermal and infrared simulation of semantic 3D models: Towards identification of urban heat islands. Infrared Physics and Technology, 105 (2020).

Bulatov, D., May, M., Strauß, E., Mancini, F., Kottler, B., Helmholz, P., 2021. Increasing level of detail of buildings for improved simulation of 4D urban digital twin. Urban Form and the Sustainable Prosperous City - The International Seminar on Urban Form (https://isuf2021.exordo.com) (accepted; publication pending).

Chen, Y.-C., Chiu, H.-W., Suc, Y.-F., Wu, Y.-C., Cheng, K.-S., 2017., Does urbanization increase diurnal land surface temperature variation? Evidence and implications. Landscape and Urban Planning, 157 (2017) 247-258.

Cohen, P., Potchter, O., Matzarakis, A., 2012. Daily and seasonal climatic conditions of green urban open spaces in the Mediterranean climate and their impact on human comfort. Building and Environment, 51 (2012) 285 - 295.

Ethell, J., 2013. Margaret Feilman 22 June 1921 - 24 September 2013. The National Trust (www.nationaltrust.org.au). Last accessed 18 June 2021.
Gallagher, R., Sigler, T., Liu, Y., 2020. 'Protect the Brisbane backyard!' (Except from subdivision for additional house construction). Australian Planner, 56:4, 278-289.

Gielen, E., Riutort-Mayol, G., Miralles i Garcia, J.L, Jiménez, J. S., P., 2021. Cost assessment of urban sprawl on municipal services using hierarchical regression. Urban Analytics and City Science, 48(2), 2021, 280-297.

Hwang, Y.H., Lum, Q.,J.,G., Chan, Y.,K.,D., 2015. Micro-scale thermal performance of tropical urban parks in Singapore. Building and Environment, 94 (2015) 467-476.

Koren, A., 2020. The missing link between growth area planning and metropolitan governance. Australian Planner, 56:2, 153157.

Kottler, B., Burkard, E., Bulatov, D., \& Haraké, L. (2019, February). Physically-based Thermal Simulation of Large Scenes for Infrared Imaging. In VISIGRAPP (1: GRAPP) (pp. 53-64).

Lee, H., Mayer, H,. 2018. Maximum extent of human heat stress reduction on building areas due to urban greening. Urban Forestry \& Urban Greening, 32 (2018) 154-167.

Liang, X., Ji, X., Guo, N., Meng, L, 2021. Assessment of urban heat islands for land use based on urban planning: a case study in the main urban area of Xuzhou City, China. Environmental Earth Sciences, 80 (2021), pp 308.

Lobaccaro, G., Acero, J.A., 2015. Comparative analysis of green actions to improve outdoor thermal comfort inside typical urban street canyons. Urban Climate, 14 (2015) 251-267.

Mahmuda, S., Webb, R., 2016. Climate adaptation and urban planning for heat islands: a case study of the Australian Capital Territory. Australian Planner, 53:2, 127-142.

Menck, C., 2014. A thematic history of government housing in Western Australia. Final Report prepared for the Department of Housing, WA. 475 pages.

Pajarola, R. (2002). Overview of quadtree-based terrain triangulation and visualization. Technical report at Information \& Computer Science Department, University of California Irvin, 2002.

Park, M., Hagishima, A., Tanimoto, J., Narita, K.-i., 2012. Effect of urban vegetation on outdoor thermal environment: Field measurement at a scale model site. Building and Environment. 56 (2012) 38-46.

Quan, S.J., Park, J., Economou, A., Lee, S., 2019. Artificial intelligence-aided design: Smart Design for sustainable city development. Urban Analytics and City Science, 46(8), 2019, $1581-1599$

Rahnam, M.R., Wyatt, R., Heydari, A., 2015. What happened from 2001 to 2011 in Melbourne? Compactness versus sprawl. Sustainable Cities and Society. 19(2015)109-120.

Vallance, S., Perkins, H.C., Moore, K., 2005. The results of making a city more compact: neighbours' interpretation of urban infill. Environment and Planning B: Planning and Design, 32 (2005), $715-733$.

Williams, S., Ahn, C., Gunc, H, Ozgirin, E., Pearce, M., Xiong, Z., 2019. Evaluating sensors for the measurement of public life: A future in image processing. Urban Analytics and City Science, 46(8), 2019, 1534-1548. 